\title{
Hypothesis Testing in Generalized Linear Models with Functional Coefficient Autoregressive Processes
}

\author{
Lei Song, ${ }^{1}$ Hongchang $\mathrm{Hu}^{1}{ }^{1}$ and Xiaosheng Cheng ${ }^{2}$ \\ ${ }^{1}$ School of Mathematics and Statistics, Hubei Normal University, Huangshi 435002, China \\ ${ }^{2}$ Department of Mathematics, Huizhou University, Huizhou 516007, China
}

Correspondence should be addressed to Hongchang Hu, retutome@163.com

Received 28 January 2012; Accepted 25 March 2012

Academic Editor: Ming Li

Copyright (C) 2012 Lei Song et al. This is an open access article distributed under the Creative Commons Attribution License, which permits unrestricted use, distribution, and reproduction in any medium, provided the original work is properly cited.

The paper studies the hypothesis testing in generalized linear models with functional coefficient autoregressive (FCA) processes. The quasi-maximum likelihood (QML) estimators are given, which extend those estimators of $\mathrm{Hu}$ (2010) and Maller (2003). Asymptotic chi-squares distributions of pseudo likelihood ratio (LR) statistics are investigated.

\section{Introduction}

Consider the following generalized linear model:

$$
y_{t}=g\left(x_{t}^{T} \beta\right)+\varepsilon_{t}, \quad t=1,2, \ldots, n,
$$

where $\beta$ is $d$-dimensional unknown parameter, $\left\{\varepsilon_{t}, t=1,2, \ldots, n\right\}$ are functional coefficient autoregressive processes given by

$$
\varepsilon_{1}=\eta_{1}, \quad \varepsilon_{t}=f_{t}(\theta) \varepsilon_{t-1}+\eta_{t}, \quad t=2,3, \ldots, n,
$$

where $\left\{\eta_{t}, t=1,2, \ldots, n\right\}$ are independent and identically distributed random variable errors with zero mean and finite variance $\sigma^{2}, \theta$ is a one-dimensional unknown parameter, and $f_{t}(\theta)$ is a real valued function defined on a compact set $\Theta$ which contains the true value $\theta_{0}$ as 
an inner point and is a subset of $R^{1}$. The values of $\theta_{0}$ and $\sigma^{2}$ are unknown. $g(\cdot)$ is a known continuous differentiable function.

Model (1.1) includes many special cases, such as an ordinary regression model (when $f_{t}(\theta) \equiv 0, g(\tau)=\tau$; see [1-7]), an ordinary generalized regression model (when $f_{t}(\theta) \equiv 0$; see [8-13]), a linear regression model with constant coefficient autoregressive processes (when $f_{t}(\theta)=\theta, g(\tau)=\tau$; see [14-16]), time-dependent and function coefficient autoregressive processes (when $g(\tau)=0$; see [17]), constant coefficient autoregressive processes (when $f_{t}(\theta)=\theta, g(\tau)=0$; see [18-20]), time-dependent or time-varying autoregressive processes (when $f_{t}(\theta)=a_{t}, g(\tau)=0$; see [21-23]), and a linear regression model with functional coefficient autoregressive processes (when $g(\tau)=\tau$; see [24]). Many authors have discussed some special cases of models (1.1) and (1.2) (see [1-24]). However, few people investigate the model (1.1) with (1.2). This paper studies the model (1.1) with (1.2). The organization of this paper is as follows. In Section 2, some estimators are given by the quasimaximum likelihood method. In Section 3, the main results are investigated. The proofs of the main results are presented in Section 4, with the conclusions and some open problems in Section 5 .

\section{The Quasi-Maximum Likelihood Estimate}

Write the "true" model as

$$
\begin{aligned}
& y_{t}=g\left(x_{t}^{T} \beta_{0}\right)+e_{t}, \quad t=1,2, \ldots, n, \\
& e_{1}=\eta_{1}, \quad e_{t}=f_{t}\left(\theta_{0}\right) e_{t-1}+\eta_{t}, \quad t=2,3, \ldots, n,
\end{aligned}
$$

where $g^{\prime}(\tau)=(d g(\tau) / d \tau) \neq 0, f_{t}^{\prime}(\theta)=\left(d f_{t}(\theta) / d \theta\right) \neq 0$. Define $\prod_{i=0}^{-1} f_{t-i}\left(\theta_{0}\right)=1$, and by $(2.2)$, we have

$$
e_{t}=\sum_{j=0}^{t-1}\left(\prod_{i=0}^{j-1} f_{t-i}\left(\theta_{0}\right)\right) \eta_{t-j}
$$

Thus $e_{t}$ is measurable with respect to the $\sigma$-field $H$ generated by $\eta_{1}, \eta_{2}, \ldots, \eta_{t}$, and

$$
E e_{t}=0, \quad \operatorname{Var}\left(e_{t}\right)=\sigma_{0}^{2} \sum_{j=0}^{t-1}\left(\prod_{i=0}^{j-1} f_{t-i}^{2}\left(\theta_{0}\right)\right)
$$

Assume at first that the $\eta_{t}$ are i.i.d. $N\left(0, \sigma^{2}\right)$, we get the log-likelihood of $y_{2}, \ldots, y_{n}$ conditional on $y_{1}$ given by

$$
\Phi_{n}=\ln L_{n}=-\frac{(n-1) \ln \sigma^{2}}{2}-\frac{\sum_{t=2}^{n}\left(\varepsilon_{t}-f_{t}(\theta) \varepsilon_{t-1}\right)^{2}}{2 \sigma^{2}}-\frac{(n-1) \ln 2 \pi}{2} .
$$


At this stage we drop the normality assumption, but still maximize (2.5) to obtain QML estimators, denoted by $\widehat{\sigma}_{n}^{2}, \widehat{\beta}_{n}, \widehat{\theta}_{n}$. The estimating equations for unknown parameters in (2.5) may be written as

$$
\begin{gathered}
\frac{\partial \Phi_{n}}{\partial \sigma^{2}}=-\frac{n-1}{2 \sigma^{2}}+\frac{1}{2 \sigma^{4}} \sum_{t=2}^{n}\left(\varepsilon_{t}-f_{t}(\theta) \varepsilon_{t-1}\right)^{2} \\
\frac{\partial \Phi_{n}}{\partial \theta}=\frac{1}{\sigma^{2}} \sum_{t=2}^{n} f_{t}^{\prime}(\theta)\left(\varepsilon_{t}-f_{t}(\theta) \varepsilon_{t-1}\right) \varepsilon_{t-1} \\
\frac{\partial \Phi_{n}}{\partial \beta_{d \times 1}}=\frac{1}{\sigma^{2}} \sum_{t=2}^{n}\left(\varepsilon_{t}-f_{t}(\theta) \varepsilon_{t-1}\right) \cdot\left(g^{\prime}\left(x_{t}^{T} \beta\right) x_{t}-f_{t}(\theta) g^{\prime}\left(x_{t-1}^{T} \beta\right) x_{t-1}\right) .
\end{gathered}
$$

Thus, $\widehat{\sigma}_{n}^{2}, \widehat{\beta}_{n}, \widehat{\theta}_{n}$ satisfy the following estimation equations

$$
\begin{gathered}
\widehat{\sigma}_{n}^{2}=\frac{1}{n-1} \sum_{t=2}^{n}\left(\widehat{\varepsilon}_{t}-f_{t}\left(\widehat{\theta}_{n}\right) \widehat{\varepsilon}_{t-1}\right)^{2}, \\
\sum_{t=2}^{n}\left(\widehat{\varepsilon}_{t}-f_{t}\left(\widehat{\theta}_{n}\right) \widehat{\varepsilon}_{t-1}\right) f_{t}^{\prime}\left(\widehat{\theta}_{n}\right) \widehat{\varepsilon}_{t-1}=0, \\
\sum_{t=2}^{n}\left(\widehat{\varepsilon}_{t}-f_{t}\left(\widehat{\theta}_{n}\right) \widehat{\varepsilon}_{t-1}\right)\left(g^{\prime}\left(x_{t}^{T} \widehat{\beta}_{n}\right) x_{t}-f_{t}\left(\widehat{\theta}_{n}\right) g^{\prime}\left(x_{t-1}^{T} \widehat{\beta}_{n}\right) x_{t-1}\right)=0,
\end{gathered}
$$

where

$$
\widehat{\varepsilon}_{t}=y_{t}-g\left(x_{t}^{T} \widehat{\beta}_{n}\right)
$$

Remark 2.1. If $g\left(x_{t}^{T} \beta\right)=x_{t}^{T} \beta$, then the above equations become the same as Hu's (see [24]). If $f_{t}(\theta)=\theta, g\left(x_{t}^{T} \beta\right)=x_{t}^{T} \beta$, then the above equations become the same as Maller's (see [15]). Thus we extend those QML estimators of Hu [24] and Maller [15].

For ease of exposition, we will introduce the following notations, which will be used later in the paper. Let $(d+1) \times 1-$ vector $\varphi=\left(\beta^{T}, \theta\right)^{T}$. Define

$$
S_{n}(\varphi)=\sigma^{2} \frac{\partial \Phi_{n}}{\partial \varphi}=\sigma^{2}\left(\frac{\partial \Phi_{n}}{\partial \beta}, \frac{\partial \Phi_{n}}{\partial \theta}\right), \quad F_{n}(\varphi)=-\sigma^{2} \frac{\partial^{2} \Phi_{n}}{\partial \varphi \partial \varphi^{T}}
$$

By (2.7), we have

$$
F_{n}(\varphi)=\left(\begin{array}{cc}
X_{n}(\varphi, \omega) & U \\
* & \sum_{t=2}^{n}\left(\left(f_{t}^{\prime 2}(\theta)+f_{t}(\theta) f_{t}^{\prime \prime}(\theta)\right) \varepsilon_{t-1}^{2}-f_{t}^{\prime \prime}(\theta) \varepsilon_{t} \varepsilon_{t-1}\right)
\end{array}\right)
$$


where the * indicates that the elements are filled in by symmetry,

$$
\begin{gathered}
X_{n}(\varphi, \omega)=-\sigma^{2}\left(\frac{\partial^{2} \Phi_{n}}{\partial \beta \partial \beta^{T}}\right), \\
U=\sum_{t=2}^{n}\left(f_{t}^{\prime}(\theta) \varepsilon_{t-1} g^{\prime}\left(x_{t}^{T} \beta\right) x_{t}+f_{t}^{\prime}(\theta) \varepsilon_{t} g^{\prime}\left(x_{t-1}^{T} \beta\right) x_{t-1}-2 f_{t}(\theta) f_{t}^{\prime}(\theta) \varepsilon_{t-1} g^{\prime}\left(x_{t-1}^{T} \beta\right) x_{t-1}\right), \\
\frac{\partial^{2} \Phi_{n}}{\partial \beta \partial \beta^{T}}=-\frac{1}{\sigma^{2}} \sum_{t=2}^{n}\left(g^{\prime}\left(x_{t}^{T} \beta\right) x_{t}-f_{t}(\theta) g^{\prime}\left(x_{t-1}^{T} \beta\right) x_{t-1}\right)\left(g^{\prime}\left(x_{t}^{T} \beta\right) x_{t}-f_{t}(\theta) g^{\prime}\left(x_{t-1}^{T} \beta\right) x_{t-1}\right)^{T} \\
+\frac{1}{\sigma^{2}} \sum_{t=2}^{n}\left(\varepsilon_{t}-f_{t}(\theta) \varepsilon_{t-1}\right)\left(g^{\prime \prime}\left(x_{t}^{T} \beta\right) x_{t} x_{t}^{T}-f_{t}(\theta) g^{\prime \prime}\left(x_{t-1}^{T} \beta\right) x_{t-1} x_{t-1}^{T}\right) .
\end{gathered}
$$

Because $\left\{e_{t-1}\right\}$ and $\left\{\eta_{t}\right\}$ are mutually independent, we have

$$
D_{n}=E\left(F_{n}\left(\varphi_{0}\right)\right)=\left(\begin{array}{cc}
X_{n}\left(\varphi_{0}\right) & 0 \\
0 & \sum_{t=2}^{n} f_{t}^{\prime 2}\left(\theta_{0}\right) E e_{t-1}^{2}
\end{array}\right)=\left(\begin{array}{cc}
X_{n}\left(\varphi_{0}\right) & 0 \\
0 & \Delta\left(\theta_{0}, \sigma_{0}\right)
\end{array}\right)
$$

where

$$
\begin{gathered}
X_{n}\left(\varphi_{0}\right)=\sum_{t=2}^{n}\left(g^{\prime}\left(x_{t}^{T} \beta_{0}\right) x_{t}-f_{t}\left(\theta_{0}\right) g^{\prime}\left(x_{t-1}^{T} \beta_{0}\right) x_{t-1}\right)\left(g^{\prime}\left(x_{t}^{T} \beta_{0}\right) x_{t}-f_{t}\left(\theta_{0}\right) g^{\prime}\left(x_{t-1}^{T} \beta_{0}\right) x_{t-1}\right)^{T} \\
\Delta\left(\theta_{0}, \sigma_{0}\right)=\sum_{t=2}^{n} f_{t}^{\prime 2}\left(\theta_{0}\right) E e_{t-1}^{2}=\sigma_{0}^{2} \sum_{t=2}^{n} f_{t}^{\prime 2}\left(\theta_{0}\right) \sum_{j=0}^{t-2}\left(\prod_{i=0}^{j-1} f_{t-i}^{2}(\theta)\right)=O(n)
\end{gathered}
$$

By (2.8) (2.7) and $E \eta_{t}=0$, we have

$$
\begin{gathered}
\sigma_{0}^{2} E\left(\left.\frac{\partial \Phi_{n}}{\partial \beta}\right|_{\beta=\beta_{0}}\right)=\sum_{t=2}^{n} E \eta_{t}\left(g^{\prime}\left(x_{t}^{T} \beta_{0}\right) x_{t}-f_{t}\left(\theta_{0}\right) g^{\prime}\left(x_{t-1}^{T} \beta_{0}\right) x_{t-1}\right)=0, \\
\sigma_{0}^{2} E\left(\left.\frac{\partial \Phi_{n}}{\partial \theta}\right|_{\theta=\theta_{0}}\right)=\sum_{t=2}^{n} f_{t}^{\prime}\left(\theta_{0}\right) E\left(\eta_{t} e_{t-1}\right)=0 .
\end{gathered}
$$

\section{Statement of Main Results}

In the section pseudo likelihood ratio (LR) statistics for various hypothesis tests of interest are derived. We consider the following hypothesis:

$$
H_{1}: g(\cdot), f(\cdot) \text { are continuous functions, and } f^{\prime(\cdot)} \neq 0, \sigma_{0}^{2}>0
$$


When the parameter space is restricted by a hypothesis $H_{0 j}, j=1,2, \ldots$, let $\widehat{\beta}_{j n}, \widehat{\theta}_{j n}, \widehat{\sigma}_{j n}^{2}$ be the corresponding QML estimators of $\beta, \theta, \sigma^{2}$, and let

$$
\widehat{L}_{j n}=-2 \Phi_{n}\left(\widehat{\beta}_{j n}, \widehat{\theta}_{j n}, \widehat{\sigma}_{j n}^{2}\right)
$$

be minus twice the log-likelihood, evaluated at the fitted parameters. Also let

$$
\begin{gathered}
\widehat{L}_{n}=-2 \Phi_{n}\left(\widehat{\beta}_{n}, \widehat{\theta}_{n}, \widehat{\sigma}_{n}^{2}\right), \\
d_{j n}=\widehat{L}_{j n}-\widehat{L}_{n}
\end{gathered}
$$

be the "deviance" statistic for testing $H_{0 j}$ against $H_{1}$. From (2.5) and (2.8),

$$
\widehat{L}_{n}=(n-1) \ln \widehat{\sigma}_{n}^{2}+(n-1)(1+\ln 2 \pi)
$$

and similarly

$$
\widehat{L}_{j n}=(n-1) \ln \widehat{\sigma}_{j n}^{2}+(n-1)(1+\ln 2 \pi)
$$

In order to obtain our results, we give some sufficient conditions as follows.

(A1) $X_{n}=\sum_{t=2}^{n} x_{t} x_{t}^{T}$ is positive definite for sufficiently large $n$ and

$$
\lim _{n \rightarrow \infty} \max _{1 \leq t \leq n} x_{t}^{T} X_{n}^{-1} x_{t}=O\left(n^{-\alpha}\right), \quad \forall \alpha \in\left(\frac{1}{2}, 1\right], \quad \lim _{n \rightarrow \infty} \sup |\lambda|_{\max }\left(X_{n}^{-1 / 2} Z_{n} X_{n}^{-T / 2}\right)<1,
$$

where $Z_{n}=(1 / 2) \sum_{t=2}^{n}\left(x_{t} x_{t-1}^{T}+x_{t-1} x_{t}^{T}\right)$ and $|\lambda|_{\max }(\cdot)$ denotes the maximum in absolute value of the eigenvalues of a symmetric matrix.

(A2) There is a constant $\alpha>0$ such that

$$
\sum_{j=1}^{t}\left(\prod_{i=0}^{j-1} f_{t-i}^{2}(\theta)\right) \leq \alpha, \quad \max _{1 \leq j \leq n}\left|\sum_{t=j+1}^{n}\left(\prod_{i=0}^{t-j-1} f_{t-i}\left(\theta_{0}\right)\right)\right| \leq \gamma .
$$

(A3) $f_{t}^{\prime}(\theta)=d f_{t}(\theta) / d \theta \neq 0$ and $f_{t}^{\prime \prime}(\theta)=d f_{t}^{\prime}(\theta) / d \theta$ exist and are bounded, and $g(\cdot)$ is twice continuously differentiable, $0<m \leq \max _{u}\left|g^{\prime}(u)\right| \leq M<\infty, 0<\widetilde{m} \leq$ $\max _{u}\left|g^{\prime \prime}(u)\right| \leq \widetilde{M}<\infty$.

Theorem 3.1. Assume (2.1), (2.2) and (A1)-(A3).

(1) Suppose $H_{01}: f_{t}(\theta)=\theta$ and $g(u)$ is a continuous function, $\sigma_{0}^{2}>0$ holds. Then

$$
d_{1 n} \stackrel{D}{\rightarrow} x_{1}^{2}, \quad n \longrightarrow \infty
$$


(2) Suppose $H_{02}: f_{t}(\theta)=\theta, g(u)=u, \sigma_{0}^{2}>0$ holds. Then

$$
d_{2 n} \stackrel{D}{\rightarrow} x_{1}^{2}, \quad n \longrightarrow \infty
$$

(3) Suppose $H_{03}: f_{t}(\theta)=\theta, g(u)=e^{u} /\left(1+e^{u}\right), \sigma_{0}^{2}>0$ holds. Then

$$
d_{3 n} \stackrel{D}{\rightarrow} x_{1}^{2}, \quad n \longrightarrow \infty
$$

\section{Proof of Theorem}

To prove Theorem 3.1, we first introduce the following lemmas.

Lemma 4.1. Suppose that (A1)-(A3) hold. Then, for all $A>0$,

$$
\sup _{\varphi \in N_{n}(A)}\left\|D_{n}^{-1 / 2} F_{n}(\varphi) D_{n}^{-T / 2}-\Phi_{n}\right\| \stackrel{P}{\rightarrow} 0, \quad n \rightarrow \infty
$$

where

$$
\begin{gathered}
\Phi_{n}=\operatorname{diag}\left(I_{d}, \frac{\sum_{t=2}^{n} f_{t}^{\prime 2}\left(\theta_{0}\right) e_{t-1}^{2}}{\Delta_{n}\left(\theta_{0}, \sigma_{0}\right)}\right), \\
N_{n}(A)=\left\{\varphi \in R^{d+1}:\left(\varphi-\varphi_{0}\right)^{T} D_{n}\left(\varphi-\varphi_{0}\right) \leq A^{2}\right\} .
\end{gathered}
$$

Proof. Similar to proof of Lemma 4.1 in $\mathrm{Hu}$ [24], here we omit.

Lemma 4.2. Suppose that (A1)-(A3) hold. Then $\widehat{\varphi}_{n} \rightarrow \varphi_{0}, \widehat{\sigma}_{n}^{2} \rightarrow \sigma_{0}^{2}$ and

$$
X_{n}\left(\beta^{*}, \beta^{* *}, \widehat{\theta}_{n}\right) \longrightarrow X_{n}\left(\varphi_{0}\right),
$$

where $\beta^{*}, \beta^{* *}$ are on the line of $\beta_{0}$ and $\widehat{\beta}_{n}$.

Proof. Similar to proof of Theorem $3.1 \mathrm{in} \mathrm{Hu} \mathrm{[24],} \mathrm{we} \mathrm{easily} \mathrm{prove} \mathrm{that} \widehat{\varphi}_{n} \rightarrow \varphi_{0}$, and $\widehat{\sigma}_{n}^{2} \rightarrow$ $\sigma_{0}^{2}$. Since (4.4) is easily proved, here we omit the proof (4.4).

Proof of Theorem 3.1. Note that $S_{n}\left(\widehat{\varphi}_{n}\right)=0$ and $F_{n}\left(\widehat{\varphi}_{n}\right)$ are nonsingular. By Taylor's expansion, we have

$$
0=S_{n}\left(\widehat{\varphi}_{n}\right)=S_{n}\left(\varphi_{0}\right)-F_{n}\left(\tilde{\varphi}_{n}\right)\left(\widehat{\varphi}_{n}-\varphi_{0}\right)
$$


where $\tilde{\varphi}_{n}=a \widehat{\varphi}_{n}+(1-a) \varphi_{0}$ for some $0 \leq a \leq 1$. Since $\widehat{\varphi}_{n} \in N_{n}(A)$, also $\tilde{\varphi}_{n} \in N_{n}(A)$. By (4.1), we have

$$
F_{n}\left(\widetilde{\varphi}_{n}\right)=D_{n}^{1 / 2}\left(\Phi_{n}+\widetilde{A}_{n}\right) D_{n}^{T / 2}
$$

Thus $\tilde{A}_{n}$ is a symmetric matrix with $\tilde{A}_{n} \stackrel{P}{\rightarrow} 0$. By (4.5) and (4.6), we have

$$
D_{n}^{T / 2}\left(\widehat{\varphi}_{n}-\varphi_{0}\right)=D_{n}^{T / 2} F_{n}^{-1}\left(\widetilde{\varphi}_{n}\right) S_{n}\left(\varphi_{0}\right)=\left(\Phi_{n}+\widetilde{A}_{n}\right)^{-1} D_{n}^{-1 / 2} S_{n}\left(\varphi_{0}\right)
$$

Let $S_{n}(\varphi), F_{n}(\varphi)$ denote $S_{n}^{(\beta)}(\varphi), S_{n}^{(\theta)}(\varphi)$, and $F_{n}^{(\beta)}(\varphi), F_{n}^{(\theta)}(\varphi)$, respectively. By $(4.7)$, we have

$$
\Phi_{n} D_{n}^{T / 2}\left(\widehat{\beta}_{n}-\beta_{0}, \widehat{\theta}_{n}-\theta_{0}\right)=D_{n}^{-1 / 2}\left(S_{n}^{(\beta)}\left(\varphi_{0}\right), S_{n}^{(\theta)}\left(\varphi_{0}\right)\right)+o_{P}(1)
$$

Note that

$$
\begin{aligned}
\Phi_{n} D_{n}^{T / 2}= & \left(\begin{array}{cc}
X_{n}^{T / 2}\left(\varphi_{0}\right) & 0 \\
0 & \frac{\left(\sum_{t=2}^{n} f_{t}^{\prime 2}\left(\theta_{0}\right) e_{t-1}^{2}\right)}{\sqrt{\Delta_{n}\left(\theta_{0}, \sigma_{0}\right)}}
\end{array}\right), \\
D_{n}^{-1 / 2} & =\left(\begin{array}{cc}
X_{n}^{-1 / 2}\left(\varphi_{0}\right) & 0 \\
0 & \frac{1}{\sqrt{\Delta_{n}\left(\theta_{0}, \sigma_{0}\right)}}
\end{array}\right) .
\end{aligned}
$$

By (2.15), (4.2) and (4.8), we get

$$
\begin{aligned}
X_{n}^{T / 2}\left(\varphi_{0}\right)\left(\widehat{\beta}_{n}-\beta_{0}\right) & =X_{n}^{-1 / 2}\left(\varphi_{0}\right) S_{n}^{(\beta)}\left(\varphi_{0}\right)+o_{P}(1) \\
& =X_{n}^{-1 / 2}\left(\varphi_{0}\right) \sum_{t=2}^{n} \eta_{t}\left(g^{\prime}\left(x_{t}^{T} \beta_{0}\right) x_{t}-f_{t}\left(\theta_{0}\right) g^{\prime}\left(x_{t-1}^{T} \beta_{0}\right) x_{t-1}\right)+o_{P}(1) \\
\sum_{t=2}^{n} f_{t}^{\prime 2}\left(\theta_{0}\right) e_{t-1}^{2}\left(\widehat{\theta}_{n}-\theta_{0}\right) & =S_{n}^{(\theta)}\left(\varphi_{0}\right)+o_{P}\left(\sqrt{\Delta_{n}\left(\theta_{0}, \sigma_{0}\right)}\right) \\
& =\sum_{t=2}^{n} f_{t}^{\prime}\left(\theta_{0}\right) \eta_{t} e_{t-1}+o_{P}\left(\sqrt{\Delta_{n}\left(\theta_{0}, \sigma_{0}\right)}\right)
\end{aligned}
$$

Note that

$$
\varepsilon_{t}=y_{t}-g\left(x_{t}^{T} \beta\right)=g^{\prime}\left(x_{t}^{T} \beta^{*}\right) x_{t}^{T}\left(\beta_{0}-\beta\right)+e_{t}
$$


By (2.1), (2.11) and (4.12), we have

$$
\widehat{\varepsilon}_{t}-f_{t}\left(\widehat{\theta}_{n}\right) \widehat{\varepsilon}_{t-1}=\left(g^{\prime}\left(x_{t}^{T} \beta^{*}\right) x_{t}^{T}-f_{t}\left(\widehat{\theta}_{n}\right) g^{\prime}\left(x_{t-1}^{T} \beta^{* *}\right) x_{t-1}^{T}\right)\left(\beta_{0}-\widehat{\beta}_{n}\right)+\left(e_{t}-f_{t}\left(\widehat{\theta}_{n}\right) e_{t-1}\right) .
$$

By (4.13) and (2.10), we have

$$
\begin{aligned}
\sum_{t=2}^{n}\left(\widehat{\varepsilon}_{t}-f_{t}\left(\widehat{\theta}_{n}\right) \widehat{\varepsilon}_{t-1}\right)^{2}= & \sum_{t=2}^{n}\left(\widehat{\varepsilon}_{t}-f_{t}\left(\widehat{\theta}_{n}\right) \widehat{\varepsilon}_{t-1}\right)\left(\left(g^{\prime}\left(x_{t}^{T} \beta^{*}\right) x_{t}^{T}-f_{t}\left(\widehat{\theta}_{n}\right) g^{\prime}\left(x_{t-1}^{T} \beta^{* *}\right) x_{t-1}^{T}\right)\right. \\
& \left.\times\left(\beta_{0}-\widehat{\beta}_{n}\right)+\left(e_{t}-f_{t}\left(\widehat{\theta}_{n}\right) e_{t-1}\right)\right) \\
= & \sum_{t=2}^{n}\left(\widehat{\varepsilon}_{t}-f_{t}\left(\widehat{\theta}_{n}\right) \widehat{\varepsilon}_{t-1}\right)\left(g^{\prime}\left(x_{t}^{T} \beta^{*}\right) x_{t}^{T}-f_{t}\left(\widehat{\theta}_{n}\right) g^{\prime}\left(x_{t-1}^{T} \beta^{* *}\right) x_{t-1}^{T}\right)\left(\beta_{0}-\widehat{\beta}_{n}\right) \\
& +\sum_{t=2}^{n}\left(\widehat{\varepsilon}_{t}-f_{t}\left(\widehat{\theta}_{n}\right) \widehat{\varepsilon}_{t-1}\right)\left(e_{t}-f_{t}\left(\widehat{\theta}_{n}\right) e_{t-1}\right) \\
= & \sum_{t=2}^{n}\left(\widehat{\varepsilon}_{t}-f_{t}\left(\widehat{\theta}_{n}\right) \widehat{\varepsilon}_{t-1}\right)\left(e_{t}-f_{t}\left(\widehat{\theta}_{n}\right) e_{t-1}\right) .
\end{aligned}
$$

By (4.13), we have

$$
\left(g^{\prime}\left(x_{t}^{T} \beta^{*}\right) x_{t}^{T}-f_{t}\left(\widehat{\theta}_{n}\right) g^{\prime}\left(x_{t-1}^{T} \beta^{* *}\right) x_{t-1}^{T}\right)\left(\beta_{0}-\widehat{\beta}_{n}\right)=\left(\widehat{\varepsilon}_{t}-f_{t}\left(\widehat{\theta}_{n}\right) \widehat{\varepsilon}_{t-1}\right)-\left(e_{t}-f_{t}\left(\widehat{\theta}_{n}\right) e_{t-1}\right) .
$$

By (4.15), we have

$$
\begin{aligned}
\sum_{t=2}^{n}( & \left.\left(g^{\prime}\left(x_{t}^{T} \beta^{*}\right) x_{t}^{T}-f_{t}\left(\widehat{\theta}_{n}\right) g^{\prime}\left(x_{t-1}^{T} \beta^{* *}\right) x_{t-1}^{T}\right)\left(\beta_{0}-\widehat{\beta}_{n}\right)\right)^{2} \\
= & \sum_{t=2}^{n}\left(\widehat{\varepsilon}_{t}-f_{t}\left(\widehat{\theta}_{n}\right) \widehat{\varepsilon}_{t-1}\right)^{2}+\sum_{t=2}^{n}\left(e_{t}-f_{t}\left(\widehat{\theta}_{n}\right) e_{t-1}\right)^{2} \\
& -2 \sum_{t=2}^{n}\left(\widehat{\varepsilon}_{t}-f_{t}\left(\widehat{\theta}_{n}\right) \widehat{\varepsilon}_{t-1}\right)\left(e_{t}-f_{t}\left(\widehat{\theta}_{n}\right) e_{t-1}\right) \\
= & \sum_{t=2}^{n}\left(e_{t}-f_{t}\left(\widehat{\theta}_{n}\right) e_{t-1}\right)^{2}-\sum_{t=2}^{n}\left(\widehat{\varepsilon}_{t}-f_{t}\left(\widehat{\theta}_{n}\right) \widehat{\varepsilon}_{t-1}\right)^{2} .
\end{aligned}
$$


By (4.14) and (4.16), we have

$$
\begin{aligned}
\sum_{t=2}^{n}\left(\widehat{\varepsilon}_{t}-f_{t}\left(\widehat{\theta}_{n}\right) \widehat{\varepsilon}_{t-1}\right)^{2}= & \sum_{t=2}^{n}\left(e_{t}-f_{t}\left(\widehat{\theta}_{n}\right) e_{t-1}\right)^{2} \\
& -\sum_{t=2}^{n}\left(\left(g^{\prime}\left(x_{t}^{T} \beta^{*}\right) x_{t}^{T}-f_{t}\left(\widehat{\theta}_{n}\right) g^{\prime}\left(x_{t-1}^{T} \beta^{* *}\right) x_{t-1}^{T}\right)\left(\beta_{0}-\widehat{\beta}_{n}\right)\right)^{2} .
\end{aligned}
$$

By (4.15), we have

$$
\begin{aligned}
\sum_{t=2}^{n}\left(\widehat{\varepsilon}_{t}\right. & \left.-f_{t}\left(\widehat{\theta}_{n}\right) \widehat{\varepsilon}_{t-1}\right)^{2} \\
= & \sum_{t=2}^{n}\left(e_{t}-f_{t}\left(\widehat{\theta}_{n}\right) e_{t-1}\right)^{2}+\sum_{t=2}^{n}\left(\left(g^{\prime}\left(x_{t}^{T} \beta^{*}\right) x_{t}^{T}-f_{t}\left(\widehat{\theta}_{n}\right) g^{\prime}\left(x_{t-1}^{T} \beta^{* *}\right) x_{t-1}^{T}\right)\left(\beta_{0}-\widehat{\beta}_{n}\right)\right)^{2} \\
& +2 \sum_{t=2}^{n}\left(e_{t}-f_{t}\left(\widehat{\theta}_{n}\right) e_{t-1}\right)\left(\left(g^{\prime}\left(x_{t}^{T} \beta^{*}\right) x_{t}^{T}-f_{t}\left(\widehat{\theta}_{n}\right) g^{\prime}\left(x_{t-1}^{T} \beta^{* *}\right) x_{t-1}^{T}\right)\left(\beta_{0}-\widehat{\beta}_{n}\right)\right) .
\end{aligned}
$$

Thus, by (4.17) and (4.18), we have

$$
\begin{aligned}
& \sum_{t=2}^{n}\left(\left(g^{\prime}\left(x_{t}^{T} \beta^{*}\right) x_{t}^{T}-f_{t}\left(\widehat{\theta}_{n}\right) g^{\prime}\left(x_{t-1}^{T} \beta^{* *}\right) x_{t-1}^{T}\right)\left(\beta_{0}-\widehat{\beta}_{n}\right)\right)^{2} \\
& \quad+\sum_{t=2}^{n}\left(e_{t}-f_{t}\left(\widehat{\theta}_{n}\right) e_{t-1}\right)\left(\left(g^{\prime}\left(x_{t}^{T} \beta^{*}\right) x_{t}^{T}-f_{t}\left(\widehat{\theta}_{n}\right) g^{\prime}\left(x_{t-1}^{T} \beta^{* *}\right) x_{t-1}^{T}\right)\left(\beta_{0}-\widehat{\beta}_{n}\right)\right)=0 .
\end{aligned}
$$

Since $\eta_{t}=e_{t}-f_{t}\left(\theta_{0}\right) e_{t-1}$, we have

$$
\begin{aligned}
\sum_{t=2}^{n}\left(e_{t}-f_{t}\left(\widehat{\theta}_{n}\right) e_{t-1}\right)^{2} & =\sum_{t=2}^{n}\left(\eta_{t}+f_{t}\left(\theta_{0}\right) e_{t-1}-f_{t}\left(\widehat{\theta}_{n}\right) e_{t-1}\right)^{2} \\
& =\sum_{t=1}^{n} \eta_{t}^{2}+\sum_{t=2}^{n}\left(f_{t}\left(\theta_{0}\right)-f_{t}\left(\widehat{\theta}_{n}\right)\right)^{2} e_{t-1}^{2}+2\left(f_{t}\left(\theta_{0}\right)-f_{t}\left(\widehat{\theta}_{n}\right)\right) \eta_{t} e_{t-1}
\end{aligned}
$$

Thus, by (4.17), (4.20) and mean value theorem, we have

$$
\begin{aligned}
(n-1) \widehat{\sigma}_{n}^{2} & =\sum_{t=2}^{n}\left(\widehat{\varepsilon}_{t}-f_{t}\left(\widehat{\theta}_{n}\right) \widehat{\varepsilon}_{t-1}\right)^{2} \\
& =\sum_{t=1}^{n} \eta_{t}^{2}+\sum_{t=2}^{n}\left(f_{t}\left(\theta_{0}\right)-f_{t}\left(\widehat{\theta}_{n}\right)\right)^{2} e_{t-1}^{2}+2\left(f_{t}\left(\theta_{0}\right)-f_{t}\left(\widehat{\theta}_{n}\right)\right) \eta_{t} e_{t-1}
\end{aligned}
$$




$$
\begin{aligned}
& -\sum_{t=2}^{n}\left(\left(g^{\prime}\left(x_{t}^{T} \beta^{*}\right) x_{t}^{T}-f_{t}\left(\widehat{\theta}_{n}\right) g^{\prime}\left(x_{t-1}^{T} \beta^{* *}\right) x_{t-1}^{T}\right)\left(\beta_{0}-\widehat{\beta}_{n}\right)\right)^{2} \\
= & \sum_{t=1}^{n} \eta_{t}^{2}+\left(\theta_{0}-\widehat{\theta}_{n}\right)^{2} \sum_{t=2}^{n} f_{t}^{\prime 2}(\widetilde{\theta}) e_{t-1}^{2}+2\left(\theta_{0}-\widehat{\theta}_{n}\right) \sum_{t=2}^{n} f_{t}^{\prime}(\widetilde{\theta}) e_{t-1} \eta_{t} \\
& -\sum_{t=2}^{n}\left(\left(g^{\prime}\left(x_{t}^{T} \beta^{*}\right) x_{t}^{T}-f_{t}\left(\widehat{\theta}_{n}\right) g^{\prime}\left(x_{t-1}^{T} \beta^{* *}\right) x_{t-1}^{T}\right)\left(\beta_{0}-\widehat{\beta}_{n}\right)\right)^{2},
\end{aligned}
$$

where $\tilde{\theta}=a \theta_{0}+(1-a) \widehat{\theta}_{n}$ for some $0 \leq a \leq 1$.

It is easy to know that

$$
\begin{aligned}
\left(\widehat{\beta}_{n}\right. & \left.-\beta_{0}\right)^{T} X_{n}\left(\varphi_{0}\right)\left(\widehat{\beta}_{n}-\beta_{0}\right) \\
& =\left(\sum_{t=2}^{n} \eta_{t} X_{n}^{-1 / 2}\left(\varphi_{0}\right)\left(g^{\prime}\left(x_{t}^{T} \beta_{0}\right) x_{t}-f_{t}\left(\theta_{0}\right) g^{\prime}\left(x_{t-1}^{T} \beta_{0}\right) x_{t-1}\right)\right)^{2}+o_{p}(1) .
\end{aligned}
$$

By Lemma 4.2 and (4.22), we have

$$
\begin{aligned}
(n-1) \widehat{\sigma}_{n}^{2}= & \sum_{t=1}^{n} \eta_{t}^{2}+\left(\theta_{0}-\widehat{\theta}_{n}\right)^{2} \sum_{t=2}^{n} f_{t}^{\prime 2}(\tilde{\theta}) e_{t-1}^{2}+2\left(\theta_{0}-\widehat{\theta}_{n}\right) \sum_{t=2}^{n} f_{t}^{\prime}(\tilde{\theta}) e_{t-1} \eta_{t} \\
& -\left(\sum_{t=2}^{n} \eta_{t} X_{n}^{-1 / 2}\left(\beta^{*}, \beta^{* *}, \widehat{\theta}_{n}\right)\left(g^{\prime}\left(x_{t}^{T} \beta_{0}\right) x_{t}-f_{t}\left(\theta_{0}\right) g^{\prime}\left(x_{t-1}^{T} \beta_{0}\right) x_{t-1}\right)\right)^{2}+o_{P}(1) \\
= & \sum_{t=1}^{n} \eta_{t}^{2}+\left(\theta_{0}-\widehat{\theta}_{n}\right)^{2} \sum_{t=2}^{n} f_{t}^{\prime 2}(\tilde{\theta}) e_{t-1}^{2}+2\left(\theta_{0}-\widehat{\theta}_{n}\right) \sum_{t=2}^{n} f_{t}^{\prime}(\tilde{\theta}) e_{t-1} \eta_{t} \\
& -\left(\sum_{t=2}^{n} \eta_{t} X_{n}^{-1 / 2}\left(\varphi_{0}\right)\left(g^{\prime}\left(x_{t}^{T} \beta_{0}\right) x_{t}-f_{t}\left(\theta_{0}\right) g^{\prime}\left(x_{t-1}^{T} \beta_{0}\right) x_{t-1}\right)\right)^{2}+o_{P}(1)
\end{aligned}
$$

Hence, by (4.11), we have

$$
\begin{aligned}
\widehat{\theta}_{n}-\theta_{0} & =\frac{\sum_{t=2}^{n} f_{t}^{\prime}\left(\theta_{0}\right) \eta_{t} e_{t-1}}{\sum_{t=2}^{n} f_{t}^{\prime 2}\left(\theta_{0}\right) e_{t-1}^{2}}+o_{P}\left(\frac{\sqrt{\Delta_{n}\left(\theta_{0}, \sigma_{0}\right)}}{\sum_{t=2}^{n} f_{t}^{\prime 2}\left(\theta_{0}\right) e_{t-1}^{2}}\right) \\
& =\frac{\sum_{t=2}^{n} f_{t}^{\prime}\left(\theta_{0}\right) \eta_{t} e_{t-1}}{\sum_{t=2}^{n} f_{t}^{\prime 2}\left(\theta_{0}\right) e_{t-1}^{2}}+o_{P}\left(\frac{1}{\sqrt{\sum_{t=2}^{n} f_{t}^{\prime 2}\left(\theta_{0}\right) e_{t-1}^{2}}}\right) .
\end{aligned}
$$


By (4.24), we have

$$
\begin{aligned}
& \left(\theta_{0}-\widehat{\theta}_{n}\right)^{2} \sum_{t=2}^{n} f_{t}^{\prime 2}(\tilde{\theta}) e_{t-1}^{2}+2\left(\theta_{0}-\widehat{\theta}_{n}\right) \sum_{t=2}^{n} f_{t}^{\prime}(\tilde{\theta}) e_{t-1} \eta_{t} \\
& =\left(\frac{\sum_{t=2}^{n} f_{t}^{\prime}\left(\theta_{0}\right) \eta_{t} e_{t-1}}{\sum_{t=2}^{n} f_{t}^{\prime 2}\left(\theta_{0}\right) e_{t-1}^{2}}+o_{P}\left(\frac{1}{\sqrt{\sum_{t=2}^{n} f_{t}^{\prime 2}\left(\theta_{0}\right) e_{t-1}^{2}}}\right)\right)^{2} \sum_{t=2}^{n} f_{t}^{\prime 2}(\tilde{\theta}) e_{t-1}^{2} \\
& +2\left(\frac{\sum_{t=2}^{n} f_{t}^{\prime}\left(\theta_{0}\right) \eta_{t} e_{t-1}}{\sum_{t=2}^{n} f_{t}^{\prime 2}\left(\theta_{0}\right) e_{t-1}^{2}}+o_{P}\left(\frac{1}{\sqrt{\sum_{t=2}^{n} f_{t}^{\prime 2}\left(\theta_{0}\right) e_{t-1}^{2}}}\right)\right) \sum_{t=2}^{n} f_{t}^{\prime}(\tilde{\theta}) e_{t-1} \eta_{t}+o_{P}(1) \\
& =\left(\frac{\sum_{t=2}^{n} f_{t}^{\prime}\left(\theta_{0}\right) \eta_{t} e_{t-1}}{\sum_{t=2}^{n} f_{t}^{\prime 2}\left(\theta_{0}\right) e_{t-1}^{2}}+o_{P}\left(\frac{1}{\sqrt{\sum_{t=2}^{n} f_{t}^{\prime 2}\left(\theta_{0}\right) e_{t-1}^{2}}}\right)\right)^{2} \sum_{t=2}^{n}\left(f_{t}^{\prime}\left(\theta_{0}\right)+o(1)\right)^{2} e_{t-1}^{2} \\
& +2\left(\frac{\sum_{t=2}^{n} f_{t}^{\prime}\left(\theta_{0}\right) \eta_{t} e_{t-1}}{\sum_{t=2}^{n} f_{t}^{\prime 2}\left(\theta_{0}\right) e_{t-1}^{2}}+o_{P}\left(\frac{1}{\sqrt{\sum_{t=2}^{n} f_{t}^{\prime 2}\left(\theta_{0}\right) e_{t-1}^{2}}}\right)\right) \\
& \cdot \sum_{t=2}^{n}\left(f_{t}^{\prime \prime}\left(\theta_{0}\right)+o(1)\right) e_{t-1} \eta_{t}+o_{P}(1) \\
& =\frac{\left(\sum_{t=2}^{n} f_{t}^{\prime}\left(\theta_{0}\right) \eta_{t} e_{t-1}\right)^{2}}{\sum_{t=2}^{n} f_{t}^{\prime 2}\left(\theta_{0}\right) e_{t-1}^{2}}-\frac{2\left(\sum_{t=2}^{n} f_{t}^{\prime}\left(\theta_{0}\right) \eta_{t} e_{t-1}\right)^{2}}{\sum_{t=2}^{n} f_{t}^{\prime 2}\left(\theta_{0}\right) e_{t-1}^{2}}+o_{P}(1) \\
& =-\frac{\left(\sum_{t=2}^{n} f_{t}^{\prime}\left(\theta_{0}\right) \eta_{t} e_{t-1}\right)^{2}}{\sum_{t=2}^{n} f_{t}^{\prime 2}\left(\theta_{0}\right) e_{t-1}^{2}}+o_{P}(1) .
\end{aligned}
$$

By Lemma 4.2, we have

$$
\begin{aligned}
(n-1) \widehat{\sigma}_{n}^{2}= & \sum_{t=1}^{n} \eta_{t}^{2}-\frac{\left(\sum_{t=2}^{n} f_{t}^{\prime}\left(\theta_{0}\right) \eta_{t} e_{t-1}\right)^{2}}{\sum_{t=2}^{n} f_{t}^{\prime 2}\left(\theta_{0}\right) e_{t-1}^{2}} \\
& -\left(\sum_{t=2}^{n} \eta_{t} X_{n}^{-1 / 2}\left(\beta^{*}, \beta^{* *}, \widehat{\theta}_{n}\right)\left(g^{\prime}\left(x_{t}^{T} \beta_{0}\right) x_{t}-f_{t}\left(\theta_{0}\right) g^{\prime}\left(x_{t-1}^{T} \beta_{0}\right) x_{t-1}\right)\right)^{2}+o_{P}(1) \\
= & \sum_{t=1}^{n} \eta_{t}^{2}-\frac{\left(\sum_{t=2}^{n} f_{t}^{\prime}\left(\theta_{0}\right) \eta_{t} e_{t-1}\right)^{2}}{\sum_{t=2}^{n} f_{t}^{\prime 2}\left(\theta_{0}\right) e_{t-1}^{2}} \\
& -\left(\sum_{t=2}^{n} \eta_{t} X_{n}^{-1 / 2}\left(\varphi_{0}\right)\left(g^{\prime}\left(x_{t}^{T} \beta_{0}\right) x_{t}-f_{t}\left(\theta_{0}\right) g^{\prime}\left(x_{t-1}^{T} \beta_{0}\right) x_{t-1}\right)\right)^{2}+o_{P}(1) .
\end{aligned}
$$


Now, we prove (3.8). By (4.12), we have

$$
\widehat{\varepsilon}_{t}(1)=y_{t}-g\left(x_{t}^{T} \widehat{\beta}_{1 n}\right)=g^{\prime}\left(x_{t}^{T} \widehat{\beta}_{1 n}^{*}\right) x_{t}^{T}\left(\beta_{0}-\widehat{\beta}_{1 n}\right)+e_{t} .
$$

Note that

$$
\varepsilon_{t}-f_{t}\left(\theta_{0}\right) \varepsilon_{t-1}=\left(g^{\prime}\left(x_{t}^{T} \beta^{*}\right) x_{t}^{T}-f_{t}\left(\theta_{0}\right) g^{\prime}\left(x_{t-1}^{T} \beta^{* *}\right) x_{t-1}^{T}\right)\left(\beta_{0}-\beta\right)+\eta_{t} .
$$

From (4.28), we have

$$
\widehat{\varepsilon}_{t}(1)-\widehat{\theta}_{1 n} \widehat{\varepsilon}_{t-1}(1)=\left(g^{\prime}\left(x_{t}^{T} \widehat{\beta}_{1 n}^{*}\right) x_{t}^{T}-\widehat{\theta}_{1 n} g^{\prime}\left(x_{t-1}^{T} \widehat{\beta}_{1 n}^{* *}\right) x_{t-1}^{T}\right)\left(\beta_{0}-\widehat{\beta}_{1 n}\right)+\eta_{t} .
$$

By (2.8) and (2.10), we have

$$
\begin{aligned}
0= & \sum_{t=2}^{n}\left(\widehat{\varepsilon}_{t}(1)-\widehat{\theta}_{1 n} \widehat{\varepsilon}_{t-1}(1)\right)\left(g^{\prime}\left(x_{t}^{T} \widehat{\beta}_{1 n}^{*}\right) x_{t}-\widehat{\theta}_{1 n} g^{\prime}\left(x_{t-1}^{T} \widehat{\beta}_{1 n}^{* *}\right) x_{t-1}\right) \\
= & \sum_{t=2}^{n}\left(g^{\prime}\left(x_{t}^{T} \widehat{\beta}_{1 n}^{*}\right) x_{t}^{T}-\widehat{\theta}_{1 n} g^{\prime}\left(x_{t-1}^{T} \widehat{\beta}_{1 n}^{* *}\right) x_{t-1}^{T}\right)\left(\beta_{0}-\widehat{\beta}_{1 n}\right)\left(g^{\prime}\left(x_{t}^{T} \widehat{\beta}_{1 n}^{*}\right) x_{t}-\widehat{\theta}_{1 n} g^{\prime}\left(x_{t-1}^{T} \widehat{\beta}_{1 n}^{* *}\right) x_{t-1}\right) \\
& +\sum_{t=2}^{n} \eta_{t}\left(g^{\prime}\left(x_{t}^{T} \widehat{\beta}_{1 n}^{*}\right) x_{t}-\widehat{\theta}_{1 n} g^{\prime}\left(x_{t-1}^{T} \widehat{\beta}_{1 n}^{* *}\right) x_{t-1}\right) \\
= & \left(\beta_{0}-\widehat{\beta}_{1 n}\right)^{T} X_{1 n}\left(\widehat{\beta}_{1 n}^{*}, \widehat{\beta}_{1 n}^{* *}, \widehat{\theta}_{1 n}\right)+\sum_{t=2}^{n} \eta_{t}\left(g^{\prime}\left(x_{t}^{T} \widehat{\beta}_{1 n}^{*}\right) x_{t}-\widehat{\theta}_{1 n} g^{\prime}\left(x_{t-1}^{T} \widehat{\beta}_{1 n}^{* *}\right) x_{t-1}\right) .
\end{aligned}
$$

From (4.30), we obtain that

$$
\widehat{\beta}_{1 n}-\beta_{0}=X_{1 n}^{-1}\left(\widehat{\beta}_{1 n}^{*}, \widehat{\beta}_{1 n}^{* *}, \widehat{\theta}_{1 n}\right) \sum_{t=2}^{n} \eta_{t}\left(g^{\prime}\left(x_{t}^{T} \widehat{\beta}_{1 n}\right) x_{t}-\widehat{\theta}_{1 n} g^{\prime}\left(x_{t-1}^{T} \widehat{\beta}_{1 n}\right) x_{t-1}\right) .
$$

By (4.29), (4.31) and Lemma 4.2, we have

$$
\begin{aligned}
(n-1) \widehat{\sigma}_{1 n}^{2}= & \sum_{t=2}^{n}\left(\widehat{\varepsilon}_{t}(1)-\widehat{\theta}_{1 n} \widehat{\varepsilon}_{t-1}(1)\right)^{2} \\
= & \sum_{t=1}^{n} \eta_{t}^{2}+\left(\beta_{0}-\widehat{\beta}_{1 n}\right)^{T} X_{1 n}\left(\widehat{\beta}_{1 n^{\prime}}^{*} \widehat{\beta}_{1 n}^{* *}, \widehat{\theta}_{1 n}\right)\left(\beta_{0}-\widehat{\beta}_{1 n}\right) \\
& +2\left(\beta_{0}-\widehat{\beta}_{1 n}\right)^{T} \sum_{t=2}^{n} \eta_{t}\left(g^{\prime}\left(x_{t}^{T} \widehat{\beta}_{1 n}^{*}\right) x_{t}-\widehat{\theta}_{1 n} g^{\prime}\left(x_{t-1}^{T} \widehat{\beta}_{1 n}^{* *}\right) x_{t-1}\right)
\end{aligned}
$$


Mathematical Problems in Engineering

$$
\begin{aligned}
& =\sum_{t=1}^{n} \eta_{t}^{2}-\left(\sum_{t=2}^{n} \eta_{t} X_{1 n}^{-1 / 2}\left(\widehat{\beta}_{1 n}^{*}, \widehat{\beta}_{1 n}^{* *}, \widehat{\theta}_{1 n}\right)\left(g^{\prime}\left(x_{t}^{T} \widehat{\beta}_{1 n}^{*}\right) x_{t}-\widehat{\theta}_{1 n} g^{\prime}\left(x_{t-1}^{T} \widehat{\beta}_{1 n}^{* *}\right) x_{t-1}\right)\right)^{2}+o_{p}(1) \\
& =\sum_{t=1}^{n} \eta_{t}^{2}-\left(\sum_{t=2}^{n} \eta_{t} X_{1 n}^{-1 / 2}\left(\varphi_{0}\right)\left(g^{\prime}\left(x_{t}^{T} \beta_{0}\right) x_{t}-\theta_{0} g^{\prime}\left(x_{t-1}^{T} \beta_{0}\right) x_{t-1}\right)\right)^{2}+o_{p} .
\end{aligned}
$$

By (3.3)-(3.5), we have

$$
d_{1 n}=\widehat{L}_{1 n}-\widehat{L}_{n}=(n-1) \ln \left(\frac{\widehat{\sigma}_{1 n}^{2}}{\widehat{\sigma}_{n}^{2}}\right)=(n-1)\left(\left(\frac{\widehat{\sigma}_{1 n}^{2}}{\widehat{\sigma}_{n}^{2}}\right)-1\right)+o_{P}(1) .
$$

Under the $H_{01}$, and by (4.26), (4.32) and (4.33), we have

$$
\begin{aligned}
\frac{(n-1)\left(\widehat{\sigma}_{1 n}^{2}-\widehat{\sigma}_{n}^{2}\right)}{\widehat{\sigma}_{n}^{2}} & =\frac{\left(\sum_{t=2}^{n} \eta_{t} e_{t-1}\right)^{2}}{\widehat{\sigma}_{n}^{2} \sum_{t=2}^{n} e_{t-1}^{2}}+o_{P}(1) \\
& =\frac{\left(\sum_{t=2}^{n} \eta_{t} e_{t-1}\right)^{2}}{\sigma_{0}^{2} \sum_{t=2}^{n} e_{t-1}^{2}}+o_{P}(1)
\end{aligned}
$$

It is easily proven that

$$
\frac{\sum_{t=2}^{n} \eta_{t} e_{t-1}}{\sigma_{0} \sqrt{\sum_{t=2}^{n} e_{t-1}^{2}}} \rightarrow N(0,1)
$$

Thus, by (4.33)-(4.35), we finish the proof of (3.8).

Next we prove (3.9). Under $\mathrm{H}_{02}: f_{t}(\theta)=\theta, g(u)=u$, and $y_{t}=x_{t}^{T} \beta_{0}+e_{t}$, we have

$$
\widehat{\varepsilon}_{t}(2)=y_{t}-x_{t}^{T} \widehat{\beta}_{2 n}=x_{t}^{T} \beta_{0}-x_{t}^{T} \widehat{\beta}_{2 n}+e_{t}=x_{t}^{T}\left(\beta_{0}-\widehat{\beta}_{2 n}\right)+e_{t}
$$

Hence

$$
\begin{aligned}
\widehat{\varepsilon}_{t}(2)-\widehat{\theta}_{2 n} \widehat{\varepsilon}_{t-1}(2) & =x_{t}^{T}\left(\beta_{0}-\widehat{\beta}_{2 n}\right)+e_{t}-\widehat{\theta}_{2 n}\left(x_{t-1}^{T}\left(\beta_{0}-\widehat{\beta}_{2 n}\right)+e_{t-1}\right) \\
& =\left(x_{t}^{T}-\widehat{\theta}_{2 n} x_{t-1}^{T}\right)\left(\beta_{0}-\widehat{\beta}_{2 n}\right)+\eta_{t} .
\end{aligned}
$$

By (2.8), (2.10), we have

$$
\begin{aligned}
0 & =\sum_{t=2}^{n}\left(\widehat{\varepsilon}_{t}(2)-\widehat{\theta}_{2 n} \widehat{\varepsilon}_{t-1}(2)\right)\left(x_{t}-\widehat{\theta}_{2 n} x_{t-1}\right) \\
& =\sum_{t=2}^{n}\left(x_{t}^{T}-\widehat{\theta}_{2 n} x_{t-1}^{T}\right)\left(\beta_{0}-\widehat{\beta}_{2 n}\right)\left(x_{t}-\widehat{\theta}_{2 n} x_{t-1}\right)+\sum_{t=2}^{n} \eta_{t}\left(x_{t}-\widehat{\theta}_{2 n} x_{t-1}\right) .
\end{aligned}
$$


From (4.38), we obtain,

$$
\widehat{\beta}_{2 n}-\beta_{0}=X_{2 n}^{-1}\left(\widehat{\theta}_{2 n}\right) \sum_{t=2}^{n} \eta_{t}\left(x_{t}-\widehat{\theta}_{2 n} x_{t-1}\right) .
$$

Thus, by (4.37), (4.39) and Lemma 4.2, we have

$$
\begin{aligned}
(n-1) \widehat{\sigma}_{2 n}^{2} & =\sum_{t=2}^{n}\left(\widehat{\varepsilon}_{t}(2)-\widehat{\theta}_{2 n} \widehat{\varepsilon}_{t-1}(2)\right)^{2} \\
& =\sum_{t=1}^{n} \eta_{t}^{2}+\left(\beta_{0}-\widehat{\beta}_{2 n}\right)^{T} X_{2 n}\left(\widehat{\theta}_{2 n}\right)\left(\beta_{0}-\widehat{\beta}_{2 n}\right)+2\left(\beta_{0}-\widehat{\beta}_{2 n}\right)^{T} \sum_{t=2}^{n} \eta_{t}\left(x_{t}-\widehat{\theta}_{2 n} x_{t-1}\right) \\
& =\sum_{t=1}^{n} \eta_{t}^{2}-\left(\sum_{t=2}^{n} \eta_{t}\left(x_{t}-\widehat{\theta}_{2 n} x_{t-1}\right)^{T}\right) X_{2 n}^{-1}\left(\widehat{\theta}_{2 n}\right)\left(\sum_{t=2}^{n} \eta_{t}\left(x_{t}-\widehat{\theta}_{2 n} x_{t-1}\right)\right) \\
& =\sum_{t=1}^{n} \eta_{t}^{2}-\left(\sum_{t=2}^{n} \eta_{t} X_{2 n}^{-1 / 2}\left(\theta_{0}\right)\left(x_{t}-\theta_{0} x_{t-1}\right)\right)^{2}+o_{p}(1) .
\end{aligned}
$$

By (3.3)-(3.5), we have

$$
d_{2 n}=\widehat{L}_{2 n}-\widehat{L}_{n}=(n-1) \ln \left(\frac{\widehat{\sigma}_{2 n}^{2}}{\widehat{\sigma}_{n}^{2}}\right)=(n-1)\left(\left(\frac{\widehat{\sigma}_{2 n}^{2}}{\widehat{\sigma}_{n}^{2}}\right)-1\right)+o_{P}(1)
$$

Under the $H_{02}$, by (4.26), (4.40), and (4.41), we obtain

$$
\begin{aligned}
\frac{(n-1)\left(\widehat{\sigma}_{2 n}^{2}-\widehat{\sigma}_{n}^{2}\right)}{\widehat{\sigma}_{n}^{2}} & =\frac{\left(\sum_{t=2}^{n} \eta_{t} e_{t-1}\right)^{2}}{\widehat{\sigma}_{n}^{2} \sum_{t=2}^{n} e_{t-1}^{2}}+o_{P}(1) \\
& =\frac{\left(\sum_{t=2}^{n} \eta_{t} e_{t-1}\right)^{2}}{\sigma_{0}^{2} \sum_{t=2}^{n} e_{t-1}^{2}}+o_{P}(1)
\end{aligned}
$$

Thus, by (4.35), (4.42), (3.9) holds.

Finally, we prove (3.10). Under $H_{03}$, we have

$$
\widehat{\varepsilon}_{t}(3)=y_{t}-\frac{e^{x_{t}^{T} \hat{\beta}_{3 n}^{*}}}{1+e^{x_{t}^{T} \hat{\beta}_{3 n}^{*}}}=\frac{e^{x_{t}^{T} \hat{\beta}_{3 n}^{*}}}{\left(1+e^{x_{t}^{T} \hat{\beta}_{3 n}^{*}}\right)^{2}} x_{t}^{T}\left(\beta_{0}-\widehat{\beta}_{3 n}\right)+e_{t} .
$$


Mathematical Problems in Engineering

15

Thus

$$
\begin{aligned}
\widehat{\varepsilon}_{t}(3)-\widehat{\theta}_{3 n} \widehat{\varepsilon}_{t-1}(3)= & \frac{e^{x_{t}^{T} \widehat{\hat{\beta}}_{3 n}^{*}}}{\left(1+e^{x_{t}^{T} \widehat{\beta}_{3 n}^{*}}\right)^{2}} x_{t}^{T}\left(\beta_{0}-\widehat{\beta}_{3 n}\right)+e_{t} \\
& -\widehat{\theta}_{3 n} \frac{e^{x_{t-1}^{T} \hat{\beta}_{3 n}^{* *}}}{\left(1+e^{x_{t-1}^{T} \widehat{\beta}_{3 n}^{* *}}\right)^{2}} x_{t-1}^{T}\left(\beta_{0}-\widehat{\beta}_{3 n}\right)-\widehat{\theta}_{3 n} e_{t-1} \\
= & \left(\frac{e^{x_{t}^{T} \widehat{\beta}_{3 n}^{*}}}{\left(1+e^{x_{t}^{T} \hat{\beta}_{3 n}^{*}}\right)^{2}} x_{t}^{T}-\widehat{\theta}_{3 n} \frac{e^{x_{t-1}^{T} \hat{\beta}_{3 n}^{* *}}}{\left(1+e^{x_{t-1}^{T} \hat{\beta}_{3 n}^{* *}}\right)^{2}} x_{t-1}^{T}\right)\left(\beta_{0}-\widehat{\beta}_{3 n}\right)+\eta_{t} .
\end{aligned}
$$

By (2.8) and (2.10), we have

$$
\begin{aligned}
& 0=\sum_{t=2}^{n}\left(\widehat{\varepsilon}_{t}(3)-\widehat{\theta}_{3 n} \widehat{\varepsilon}_{t-1}(3)\right)\left(\frac{e^{x_{t}^{T} \widehat{\hat{\beta}}_{3 n}^{*}}}{\left(1+e^{x_{t}^{T} \widehat{\beta}_{3 n}^{*}}\right)^{2}} x_{t}-\widehat{\theta}_{3 n} \frac{e^{x_{t-1}^{T} \hat{\beta}_{3 n}^{* *}}}{\left(1+e^{x_{t-1}^{T} \hat{\beta}_{3 n}^{* *}}\right)^{2}} x_{t-1}\right) \\
& =\sum_{t=2}^{n}\left(\frac{e^{x_{t}^{T} \widehat{\hat{\beta}}_{3 n}^{*}}}{\left(1+e^{x_{t}^{T} \hat{\beta}_{3 n}^{*}}\right)^{2}} x_{t}^{T}-\widehat{\theta}_{3 n} \frac{e^{x_{t-1}^{T} \hat{\beta}_{3 n}^{* *}}}{\left(1+e^{x_{t-1}^{T} \hat{\beta}_{3 n}^{* *}}\right)^{2}} x_{t-1}^{T}\right)\left(\beta_{0}-\widehat{\beta}_{3 n}\right) \\
& \times\left(\frac{e^{x_{t}^{T} \hat{\beta}_{3 n}^{*}}}{\left(1+e^{x_{t}^{T} \hat{\beta}_{3 n}^{*}}\right)^{2}} x_{t}-\widehat{\theta}_{3 n} \frac{e^{x_{t-1}^{T} \hat{\beta}_{3 n}^{* *}}}{\left(1+e^{x_{t-1}^{T} \hat{\beta}_{3 n}^{* *}}\right)^{2}} x_{t-1}\right) \\
& +\sum_{t=2}^{n} \eta_{t}\left(\frac{e^{x_{t}^{T} \widehat{\beta}_{3 n}^{*}}}{\left(1+e^{x_{t}^{T} \hat{\beta}_{3 n}^{*}}\right)^{2}} x_{t}-\widehat{\theta}_{3 n} \frac{e^{x_{t-1}^{T} \hat{\beta}_{3 n}^{* *}}}{\left(1+e^{x_{t-1}^{T} \hat{\beta}_{3 n}^{* *}}\right)^{2}} x_{t-1}\right) \\
& =\left(\beta_{0}-\widehat{\beta}_{3 n}\right)^{T} X_{3 n}\left(\widehat{\beta}_{3 n}^{*}, \widehat{\beta}_{3 n}^{* *}, \widehat{\theta}_{3 n}\right)+\sum_{t=2}^{n} \eta_{t}\left(\frac{e^{x_{t}^{T} \widehat{\beta}_{3 n}^{*}}}{\left(1+e^{x_{t}^{T} \hat{\beta}_{3 n}^{*}}\right)^{2}} x_{t}-\widehat{\theta}_{3 n} \frac{e^{x_{t-1}^{T} \hat{\beta}_{3 n}^{* *}}}{\left(1+e^{x_{t-1}^{T} \hat{\beta}_{3 n}^{* *}}\right)^{2}} x_{t-1}\right) .
\end{aligned}
$$

From (4.45), we obtain

$$
\widehat{\beta}_{3 n}-\beta_{0}=X_{3 n}^{-1}\left(\widehat{\beta}_{3 n}^{*}, \widehat{\beta}_{3 n}^{* *}, \widehat{\theta}_{3 n}\right) \sum_{t=2}^{n} \eta_{t}\left(\frac{e^{x_{t}^{T} \widehat{\beta}_{3 n}^{*}}}{\left(1+e^{x_{t}^{T} \widehat{\beta}_{3 n}^{*}}\right)^{2}} x_{t}-\widehat{\theta}_{3 n} \frac{e^{x_{t-1}^{T} \hat{\beta}_{3 n}^{* *}}}{\left(1+e^{x_{t-1}^{T} \widehat{\beta}_{3 n}^{* *}}\right)^{2}} x_{t-1}\right) .
$$


By (4.44), (4.46) and Lemma 4.2, we have

$$
\begin{aligned}
& (n-1) \widehat{\sigma}_{3 n}^{2}=\sum_{t=2}^{n}\left(\widehat{\varepsilon}_{t}(3)-\widehat{\theta}_{3 n} \widehat{\varepsilon}_{t-1}(3)\right)^{2} \\
& =\sum_{t=1}^{n} \eta_{t}^{2}+\left(\beta_{0}-\widehat{\beta}_{3 n}\right)^{T} X_{3 n}\left(\widehat{\beta}_{3 n}^{*}, \widehat{\beta}_{3 n}^{* *}, \widehat{\theta}_{3 n}\right)\left(\beta_{0}-\widehat{\beta}_{3 n}\right) \\
& +2\left(\beta_{0}-\widehat{\beta}_{3 n}\right)^{T} \sum_{t=2}^{n} \eta_{t}\left(\frac{e^{x_{t}^{T} \hat{\beta}_{3 n}^{*}}}{\left(1+e^{x_{t}^{T} \hat{\beta}_{3 n}^{*}}\right)^{2}} x_{t}-\widehat{\theta}_{3 n} \frac{e^{x_{t-1}^{T} \widehat{\beta}_{3 n}^{* *}}}{\left(1+e^{x_{t-1}^{T} \hat{\beta}_{3 n}^{* *}}\right)^{2}} x_{t-1}\right) \\
& =\sum_{t=1}^{n} \eta_{t}^{2}-\left(\sum_{t=2}^{n} \eta_{t} X_{3 n}^{-1 / 2}\left(\widehat{\beta}_{3 n}^{*}, \widehat{\beta}_{3 n^{\prime}}^{* *} \widehat{\theta}_{3 n}\right)\right. \\
& \left.\times\left(\frac{e^{x_{t}^{T} \hat{\beta}_{3 n}^{*}}}{\left(1+e^{x_{t}^{T} \hat{\beta}_{3 n}^{*}}\right)^{2}} x_{t}^{T}-\widehat{\theta}_{3 n} \frac{e^{x_{t-1}^{T} \hat{\beta}_{3 n}^{* *}}}{\left(1+e^{x_{t-1}^{T} \hat{\beta}_{3 n}^{* *}}\right)^{2}} x_{t-1}^{T}\right)\right)^{2} \\
& =\sum_{t=1}^{n} \eta_{t}^{2}-\left(\sum_{t=2}^{n} \eta_{t} X_{3 n}^{-1 / 2}\left(\varphi_{0}\right)\left(\frac{e^{x_{t}^{T} \beta_{0}}}{\left(1+e^{x_{t}^{T} \beta_{0}}\right)^{2}} x_{t}-\theta_{0} \frac{e^{x_{t-1}^{T} \beta_{0}}}{\left(1+e^{x_{t-1}^{T} \beta_{0}}\right)^{2}} x_{t-1}\right)\right)^{2}+o_{p}(1) .
\end{aligned}
$$

By (3.3)-(3.5), we know that

$$
d_{3 n}=\widehat{L}_{3 n}-\widehat{L}_{n}=(n-1) \ln \left(\frac{\widehat{\sigma}_{3 n}^{2}}{\widehat{\sigma}_{n}^{2}}\right)=(n-1)\left(\left(\frac{\widehat{\sigma}_{3 n}^{2}}{\widehat{\sigma}_{n}^{2}}\right)-1\right)+o_{P}(1)
$$

Under the $H_{03}$, by (4.26), (4.47) and (4.48), we have

$$
\frac{(n-1)\left(\widehat{\sigma}_{3 n}^{2}-\widehat{\sigma}_{n}^{2}\right)}{\widehat{\sigma}_{n}^{2}}=\frac{\left(\sum_{t=2}^{n} \eta_{t} e_{t-1}\right)^{2}}{\sigma_{0}^{2} \sum_{t=2}^{n} e_{t-1}^{2}}+o_{P}(1)
$$

Thus, (3.10) follows from (4.48), (4.49), and (4.35). Therefore, we complete the proof of Theorem 3.1.

\section{Conclusions and Open Problems}

In the paper, we consider the generalized linear mode with FCA processes, which includes many special cases, such as an ordinary regression model, an ordinary generalized regression model, a linear regression model with constant coefficient autoregressive processes, timedependent and function coefficient autoregressive processes, constant coefficient autoregressive processes, time-dependent or time-varying autoregressive processes, and a linear 
regression model with functional coefficient autoregressive processes. And then we obtain the QML estimators for some unknown parameters in the generalized linear mode model and extend some estimators. At last, we use pseudo LR method to investigate three hypothesis tests of interest and obtain the asymptotic chi-squares distributions of statistics.

However, several lines of future work remain open.

(1) It is well known that a conventional time series can be regarded as the solution to a differential equation of integer order with the excitation of white noise in mathematics, and a fractal time series can be regarded as the solution to a differential equation of fractional order with a white noise in the domain of stochastic processes (see [25]). In the paper, $\left\{\varepsilon_{t}\right\}$ is a conventional nonlinear time series. We may investigate some hypothesis tests by pseudo LR method when the $\left\{\varepsilon_{t}\right\}$ is a fractal time series (the idea is given by an anonymous reviewer). In particular, we assume that

$$
\sum_{i=0}^{p} a_{p-i} D^{v_{i}} \varepsilon_{t}=\eta_{t}
$$

where $v_{p}, v_{p-1}, \ldots, v_{0}$ is strictly decreasing sequence of nonnegative numbers, $a_{i}$ is a constant sequence, and $D^{v}$ is the Riemann-Liouville integral operator of order $v>0$ given by

$$
D^{v} h(t)=\frac{1}{\Gamma(v)} \int_{0}^{t}(t-u)^{v-1} h(u) d u
$$

where $\Gamma$ is the Gamma function, and $h(t)$ is a piecewise continuous on $(0, \infty)$ and integrable on any finite subinterval of $[0, \infty)$ (See $[25,26]$ ). Fractal time series may have a heavytailed probability distribution function and has been applied various fields of sciences and technologies (see [25, 27-32]). Thus it is very significant to investigate various regression models with fractal time series errors, including regression model (1.1) with (5.1).

(2) We maybe investigate the others hypothesis tests, for example:

$$
\begin{aligned}
& H_{04}: f_{t}(\theta)=0, g(u)=u, \sigma_{0}^{2}>0 ; \\
& H_{05}: f_{t}(\theta)=\theta, g(u)=0, \sigma_{0}^{2}>0 ; \\
& H_{06}: f_{t}(\theta)=0, g(u)=e^{u} /\left(1+e^{u}\right), \sigma_{0}^{2}>0 ; \\
& H_{07}: f_{t}(\theta)=a_{t} \text { and } g(u) \text { is a continuous function, } \sigma_{0}^{2}>0 ; \\
& H_{08}: f_{t}(\theta)=a_{t}, g(u)=u, \sigma_{0}^{2}>0 ; \\
& H_{09}: f_{t}(\theta)=a_{t}, g(u)=e^{u} /\left(1+e^{u}\right), \sigma_{0}^{2}>0 .
\end{aligned}
$$

\section{Acknowledgments}

The authors would like to thank the anonymous referees for their valuable comments which have led to this much improved version of the paper. The paper was supported by Scientific Research Item of Department of Education, Hubei (no. D20112503), Scientific Research Item of Ministry of Education, China (no. 209078), and Natural Science Foundation of China (no. 11071022, 11101174). 


\section{References}

[1] Z. D. Bai and M. Guo, "A paradox in least-squares estimation of linear regression models," Statistics E Probability Letters, vol. 42, no. 2, pp. 167-174, 1999.

[2] Y. Li and H. Yang, "A new stochastic mixed ridge estimator in linear regression model," Statistical Papers, vol. 51, no. 2, pp. 315-323, 2010.

[3] A. E. Hoerl and R. W. Kennard, "Ridge regression: Biased estimation for nonorthogonal problems," Technometrics, vol. 12, pp. 55-67, 1970.

[4] X. Chen, "Consistency of LS estimates of multiple regression under a lower order moment condition," Science in China Series A, vol. 38, no. 12, pp. 1420-1431, 1995.

[5] T. W. Anderson and J. B. Taylor, "Strong consistency of least squares estimates in normal linear regression," The Annals of Statistics, vol. 4, no. 4, pp. 788-790, 1976.

[6] G. González-Rodríguez, A. Blanco, N. Corral, and A. Colubi, "Least squares estimation of linear regression models for convex compact random sets," Advances in Data Analysis and Classification, vol. 1, no. 1, pp. 67-81, 2007.

[7] H. Cui, "On asymptotics of t-type regression estimation in multiple linear model," Science in China Series A, vol. 47, no. 4, pp. 628-639, 2004.

[8] M. Q. Wang, L. X. Song, and X. G. Wang, "Bridge estimation for generalized linear models with a diverging number of parameters," Statistics $\mathcal{E}$ Probability Letters, vol. 80, no. 21-22, pp. 1584-1596, 2010.

[9] L. C. Chien and T. S. Tsou, "Deletion diagnostics for generalized linear models using the adjusted Poisson likelihood function," Journal of Statistical Planning and Inference, vol. 141, no. 6, pp. 2044-2054, 2011.

[10] L. Fahrmeir and H. Kaufmann, "Consistency and asymptotic normality of the maximum likelihood estimator in generalized linear models," The Annals of Statistics, vol. 13, no. 1, pp. 342-368, 1985.

[11] Z. H. Xiao and L. Q. Liu, "Laws of iterated logarithm for quasi-maximum likelihood estimator in generalized linear model," Journal of Statistical Planning and Inference, vol. 138, no. 3, pp. 611-617, 2008.

[12] Y. Bai, W. K. Fung, and Z. Zhu, "Weighted empirical likelihood for generalized linear models with longitudinal data," Journal of Statistical Planning and Inference, vol. 140, no. 11, pp. 3446-3456, 2010.

[13] S. G. Zhao and Y. Liao, "The weak consistency of maximum likelihood estimators in generalized linear models," Science in China A, vol. 37, no. 11, pp. 1368-1376, 2007.

[14] P. Pere, "Adjusted estimates and Wald statistics for the AT(1) model with constant," Journal of Econometrics, vol. 98, no. 2, pp. 335-363, 2000.

[15] R. A. Maller, "Asymptotics of regressions with stationary and nonstationary residuals," Stochastic Processes and Their Applications, vol. 105, no. 1, pp. 33-67, 2003.

[16] W. A. Fuller, Introduction to Statistical Time Series, John Wiley \& Sons, New York, NY, USA, 2nd edition, 1996.

[17] G. H. Kwoun and Y. Yajima, "On an autoregressive model with time-dependent coefficients," Annals of the Institute of Statistical Mathematics, vol. 38, no. 2, pp. 297-309, 1986.

[18] J. S. White, "The limiting distribution of the serial correlation coefficient in the explosive case," Annals of Mathematical Statistics, vol. 29, pp. 1188-1197, 1958.

[19] J. S. White, "The limiting distribution of the serial correlation coefficient in the explosive case-II," Annals of Mathematical Statistics, vol. 30, pp. 831-834, 1959.

[20] J. D. Hamilton, Time Series Analysis, Princeton University Press, Princeton, NJ, USA, 1994.

[21] F. Carsoule and P. H. Franses, "A note on monitoring time-varying parameters in an autoregression," International Journal for Theoretical and Applied Statistics, vol. 57, no. 1, pp. 51-62, 2003.

[22] R. Azrak and G. Mélard, "Asymptotic properties of quasi-maximum likelihood estimators for ARMA models with time-dependent coefficients," Statistical Inference for Stochastic Processes, vol. 9, no. 3, pp. 279-330, 2006.

[23] R. Dahlhaus, "Fitting time series models to nonstationary processes," The Annals of Statistics, vol. 25, no. 1, pp. 1-37, 1997.

[24] H. Hu, "QML estimators in linear regression models with functional coefficient autoregressive processes," Mathematical Problems in Engineering, vol. 2010, Article ID 956907, 30 pages, 2010.

[25] M. Li, "Fractal time series-a tutorial review," Mathematical Problems in Engineering, vol. 2010, Article ID 157264, 26 pages, 2010.

[26] Y. S. Mishura, Stochastic Calculus for Fractional Brownian Motion and Related Processes, vol. 1929 of Lecture Notes in Mathematics, Springer, Berlin, Germany, 2008. 
[27] M. Li and W. Zhao, "Visiting power laws in cyber-physical networking systems," Mathematical Problems in Engineering, vol. 2012, Article ID 302786, 13 pages, 2012.

[28] M. Li, C. Cattani, and S. Y. Chen, "Viewing sea level by a one-dimensional random function with long memory," Mathematical Problems in Engineering, vol. 2011, Article ID 654284, 13 pages, 2011.

[29] C. Cattani, "Fractals and hidden symmetries in DNA," Mathematical Problems in Engineering, vol. 2010, Article ID 507056, 31 pages, 2010.

[30] J. Lévy-Véhel and E. Lutton, Fractals in Engineering, Springer, 1st edition, 2005.

[31] V. Pisarenko and M. Rodkin, Heavy-Tailed Distributions in Disaster Analysis, Springer, 2010.

[32] H. Sheng, Y.-Q. Chen, and T.-S. Qiu, Fractional Processes and Fractional Order Signal Processing, Springer, 2012. 


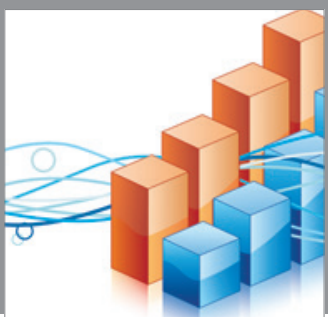

Advances in

Operations Research

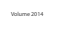

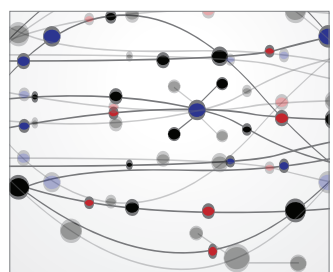

\section{The Scientific} World Journal
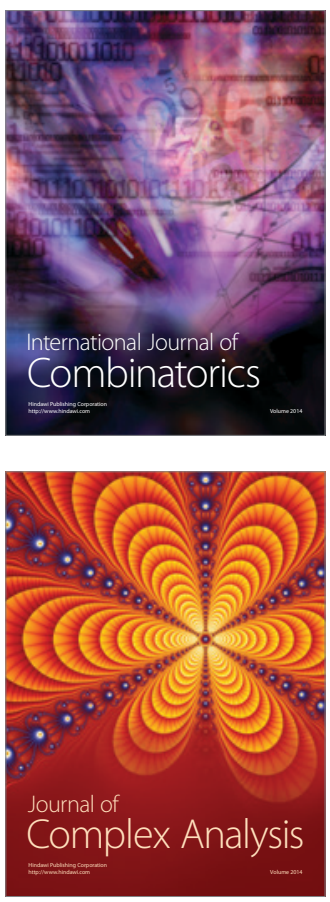

International Journal of

Mathematics and

Mathematical

Sciences
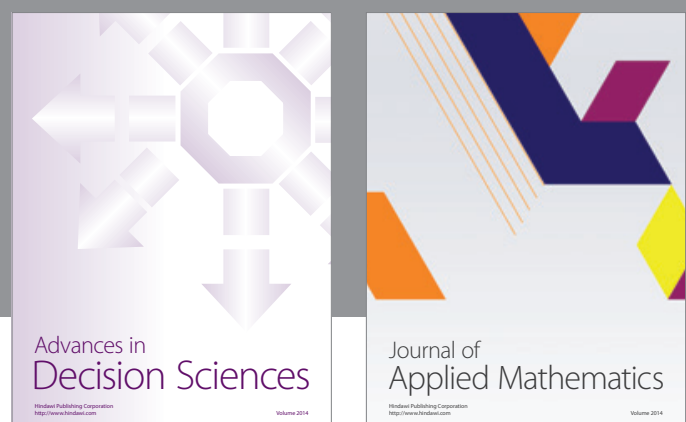

Journal of

Applied Mathematics
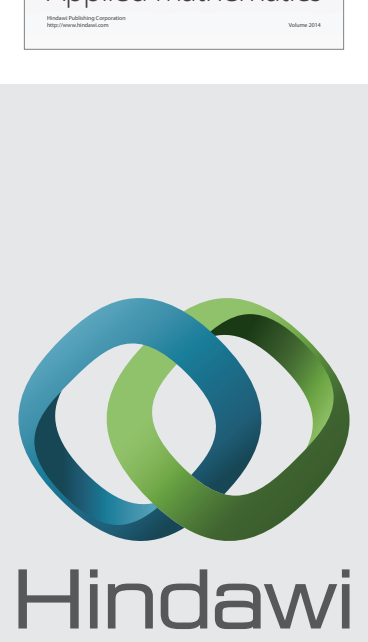

Submit your manuscripts at http://www.hindawi.com
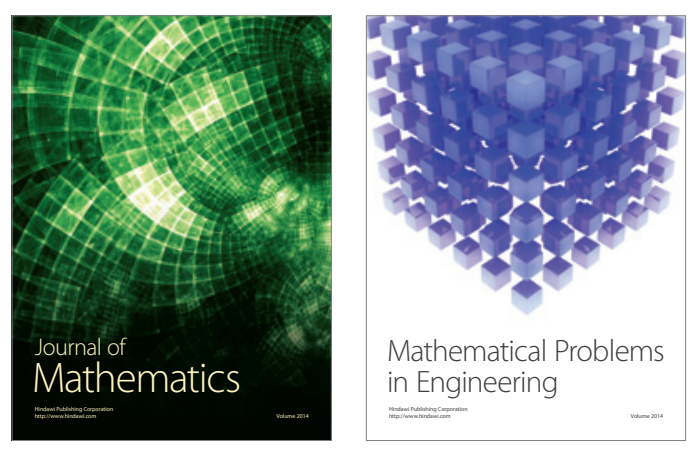

Mathematical Problems in Engineering
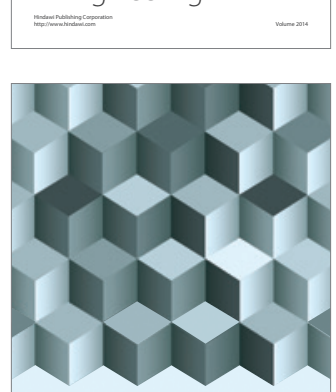

Journal of

Function Spaces
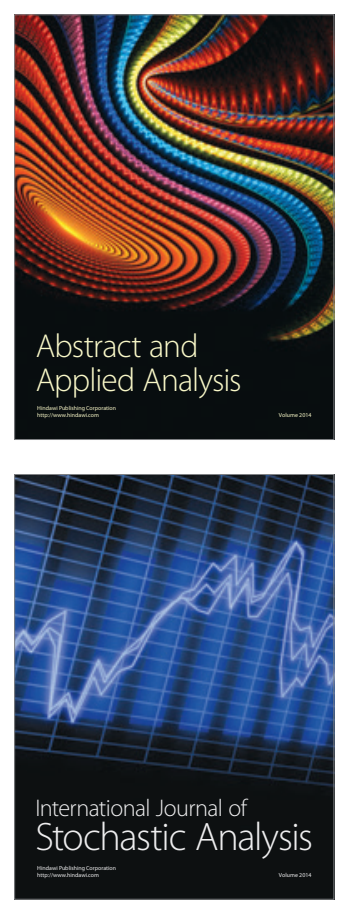

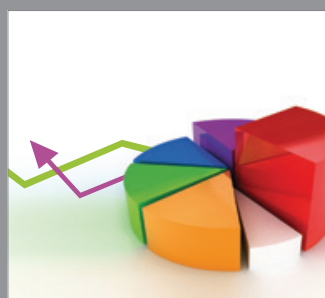

ournal of

Probability and Statistics

Promensencen
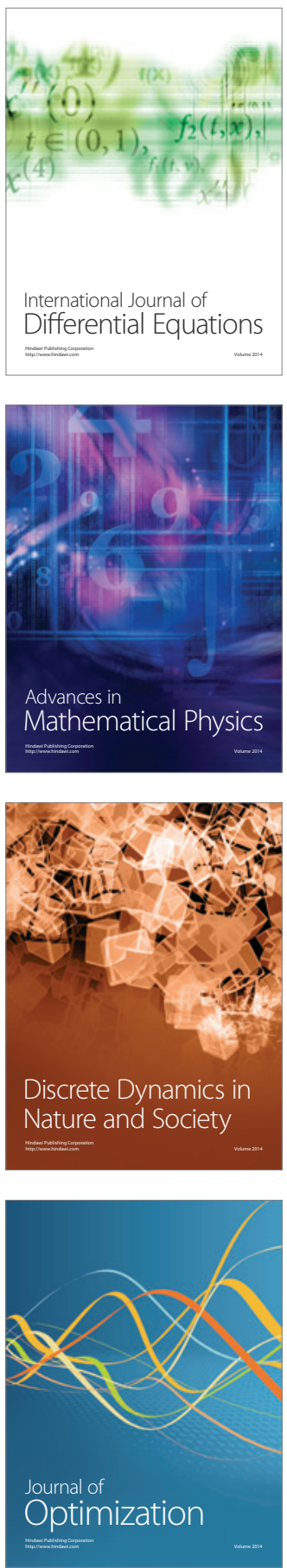